\title{
Anemia e Desnutrição em Escolares da Rede Pública do Município de Osasco, São Paulo, Brasil'
}

\author{
Anemia and Malnutrition in Children at Public Schools in Osasco, \\ São Paulo, Brazil
}

\author{
Maria Lúcia R. Stefanini ; Célia Colli ; Barbara Regina Lerner² ; \\ Doris Lucia M. Lei ; Sandra P. Chaves² ; Marcello S. Di Pietro² ; \\ Antonio Altair M. Oliveira ${ }^{3}$; Sophia C. Szarfarc ${ }^{4}$
}

STEFANINI, M. L. R.; COLLI, C.; LERNER, B. R.; LEI, D. L. M.; CHAVES, S. P.; DI PIETRO, M. S.; OLIVEIRA, A. A. M. \& SZARFARC, S. C. Anemia and Malnutrition in Children at Public Schools in Osasco, São Paulo, Brazil. Cad. Saúde Públ., Rio de Janeiro, 11 (3): 439-447, Jul/Sep, 1995.

The authors studied a sample of students entering the first grade in the Osasco public school system in order to determine both the prevalence of anemia and nutritional status. Osasco is part of the Greater São Paulo Metropolitan Area. Diagnosis of anemia was made through the hemoglobin concentration of blood from digital puncture. World Health Organization (WHO) levels were used to define anemia. Nutritional Status assessment. was made through weight/ age and height/age indices, using $Z$ score distribution and the National Center for Health Statistics (NCHS) reference levels. Prevalence of anemia was $51 \%$. Prevalence levels varied according to the schools' geographic location: $56.9 \%$ in peripheral neighborhoods and $41.7 \%$ in central areas. Children with illiterate parents had a higher prevalence of this condition. Risk of anemia was higher for children who were over eight years of age when entering the first grade. Acute malnutrition was not found. Prevalence was higher than expected and points to the urgent need to establish an anemia control program for schoolchildren in this population.

Key words: Iron Deficiency; Anemia; Nutritional Anemia; Undernutrition; Schoolchildren.

\section{INTRODUÇÃO}

Em 1980, a Organização Mundial da Saúde (OMS) estimava a existência de 700 milhões de indivíduos anêmicos em todo o mundo, com importantes repercussões sobre seu desenvolvimento físico e mental. Nessa época, nos países subdesenvolvidos, mais da metade das crianças

\footnotetext{
${ }^{1}$ Trabalho realizado com o auxílio financeiro do Instituto Nacional de Alimentação e Nutrição, Conv. 03/92.

${ }^{2}$ Instituto de Saúde, Secretaria da Saúde de São Paulo. Rua Santo Antonio, 590, São Paulo, SP, 01314-000, Brasil.

${ }^{3}$ Faculdade de Ciências Farmacêuticas, Universidade de São Paulo. Av. Prof: Lineu Prestes, 580, São Paulo, SP, 05508-900, Brasil.

${ }^{4}$ Departamento de Nutrição, Faculdade de Saúde Pública, Universidade de São Paulo. Av. Dr. Arnaldo, 715, São Paulo, SP, 01246-904, Brasil.
}

de até 4 anos e das gestantes eram anêmicas (DeMaeyer et al., 1989).

A deficiência de ferro, mesmo na forma moderada, representa um considerável agravo à saúde, estando associada a prejuízos na capacidade produtiva dos indivíduos, no desenvolvimento cognitivo e na imunocompetência (Moyses, 1979).

A anemia nutricional é um inquestionável problema de saúde pública no Brasil. Becker \& Lechtig (1986), através de dados da Divisão Nacional de Epidemiologia, concluiram que cerca de 14 mil óbitos (1,3\% do total) entre a população infantil brasileira são causados direta ou indiretamente pela anemia, sendo esta responsável também por cerca da metade dos óbitos em menores de 5 anos e em mulheres em idade fértil na região nordeste do Brasil.

A maioria dos estudos epidemiológicos sobre anemia no nosso país refere-se exclusiva- 
mente a crianças menores de 6 anos (Lira et al., 1985; Sigulem et al., 1978) e a gestantes (Salzano et al., 1980; Szarfarc, 1974, 1985a), considerados os grupos de maior vulnerabilidade, ou a usuários de serviços de saúde (Monteiro \& Szarfarc, 1987; Salzano et al., 1985; Szarfarc, 1985b).

Na cidade de São Paulo, em 1974, Sigulem et al. (1978) encontraram prevalência de $22,7 \%$ de anemia em crianças com idade entre 6 e 60 meses, com elevadas freqüências em menores de 1 ano $(30,2 \%)$ e em crianças no $2^{\circ}$ ano de vida (34,9\%n). Em 1984, Monteiro \& Szarfarc (1987), em outro estudo populacional no município de São Paulo, que permitiu a análise comparativa, com aquele efetuado por Sigulem et al. em 1974, encontraram 35,5\% de anêmicos. Em dez anos observou-se, pois, um aumento de mais de $50 \%$ em relação à totalidade de casos em crianças menores de 5 anos, sendo que, as maiores prevalências foram encontradas em crianças entre 6 e 24 meses de idade. Neste último estudo encontrou-se $53,7 \%$ de anêmicos entre crianças de 6 a 11 meses e $58,1 \%$ nas de 12 a 24 meses.

Com relação aos escolares, há poucos estudos sobre anemia. Os escassos dados, contudo, sugerem alta prevalência em crianças dessa faixa etária. Cesar (1990), em pesquisa realizada em uma escola do município de São Paulo, encontrou uma prevalência de $60 \%$ de anêmicos e de $36 \%$ de desnutridos entre as crianças de $1^{\underline{a}}$ a $4^{\underline{a}}$ séries. Nesse estudo, observou-se que, embora a desnutrição e a anemia não estivessem associadas, a presença de uma ou de outra se relacionou com a repetência e com a idade inadequada da criança na série escolar. A evasão escolar ocorreu de forma preocupante: formaram-se oito classes de $1^{\mathrm{a}}$ série e a cada série escolar, perdeu-se uma classe inteira, chegando a apenas cinco classes na $4^{\mathrm{a}}$ série. Este fato destaca a deficiência de ferro como importante problema presente nessa faixa de idade necessitando atenção dos pesquisadores.

$\mathrm{O}$ presente trabalho faz parte de um projeto mais amplo sobre intervenção nutricional com alimentos enriquecidos com ferro. Com a finalidade inicial de verificar a dimensão do problema, desenhou-se um estudo para estimar a prevalência e o risco da anemia e conhecer o estado nutricional em crianças ingressantes na rede escolar de um município da Região Metropolitana de São Paulo.

\section{METODOLOGIA}

A população de estudo foi constituída pelos escolares ingressantes, no ano de 1991, nas escolas públicas de Osasco, município que faz parte da Região Metropolitana de São Paulo e possui características socioeconômicas e demográficas que reproduzem o perfil encontrado na maioria dos municípios dessa região.

Em Osasco há 102 escolas, sendo 83 (81\%) públicas e 19 (19\%) particulares. Optou-se em estudar apenas a prevalência de anemia em escolas públicas, uma vez que nestas poderiam ser implantadas e avaliadas propostas de intervenção nutricional através da merenda escolar.

A amostragem utilizada foi casual sistemática, com unidade amostral "classe escolar" e fração $10 \%$ do universo. As 83 escolas públicas tinham 395 classes de $1^{\text {a }}$ série com um total de 14088 alunos ingressantes, com 35 alunos por classe em média.

A ordenação das classes levou em conta um estudo de 1989 que mediu a prevalência de déficit de crescimento (estatura inferior a 2 desvios-padrão do valor esperado para idade, utilizando como referência a população do NCHS/ OMS) em alunos ingressantes no $1^{\circ}$ grau de escolas públicas e privadas do município de Osasco (Monteiro et al., 1989). Nesse estudo identificaram-se duas áreas consideradas homogêneas para a prevalência de déficit de crescimento: área central com prevalência de déficit de crescimento inferior a 6\% e área periférica, apresentando a pior situação, com prevalência variando de $6 \%$ a 16\%. Conhecendo-se a prevalência de déficit por escola conforme resultado desse Censo de Estatura, as 83 escolas públicas foram ordenadas de forma crescente, permitindo assim que todas as escolas tivessem a mesma chance de entrar na amostra. A partir dessa ordenação, foram listadas as 395 classes de $1^{\text {a }}$ série e procedeu-se ao sorteio proporcional por região. Foram sorteadas 40 classes (1367 alunos ingressantes), sendo 16 localizadas na região central e 24 na região periférica.

O diagnóstico de anemia foi feito através da dosagem de hemoglobina $(\mathrm{Hb})$ pelo método da 
cianometahemoglobina, com padrão artificial de calibração, empregando-se o valor-limite de concentração de $\mathrm{Hb}$ inferior a $12 \mathrm{~g} / \mathrm{dl}$, estabelecido pela OMS (1975).

As medidas antropométricas utilizadas foram o peso e a altura. O peso foi obtido em balança eletrônica e a altura com o use de estadiômetro portátil e prumo, segundo técnica recomendada por Jelliffe (1968).

Para avaliação das medidas antropométricas foi adotada a população de referência do National Center for Health Statistics (NCHS), conforme recomendação da OMS (WHO, 1986). Os indicadores de peso e de estatura para idade e sexo e de peso para altura foram expressos em escore 2, ou seja, unidades de desvio-padrão distantes da medida esperada para a população de referência.

Data de nascimento e sexo foram colhidos da ficha de matrícula dos escolares, para o cálculo da idade, no momento da colheita de sangue e de medidas. Através de carta dirigida aos pais obteve-se informação da escolaridade dos mesmos e a autorização para a colheita de sangue por punção digital.

Utilizou-se o indicador "nível de escolaridade do pai" para a classificação socioeconômica, adotando-se as seguintes categorias para definir a escolaridade: analfabeto - não estudou e não sabe ler/escrever; primário in- completo - estudou de 1 a 3 anos; primário completo - estudou de 4 a 7 anos; ginásio completo - concluiu o primeiro grau a/ou mais anos de estudo.

Para a análise de associação, foi utilizado o teste do qui quadrado $\left(\div^{2}\right)$, adotando-se nível de significância igual a 5\%. Para a comparação de duas médias, foi utilizado o teste "t" de Student. Foi estimada a razão de prevalência (RP) por ponto e com intervalo de confiança (IC) de $95 \%$.

\section{RESULTADOS}

\section{Característica da População}

A amostra estudada foi constituída por 1033 crianças ingressantes na primeira série do primeiro grau de escolas públicas do Município de Osasco, Estado de São Paulo.

Embora a listagem das classes sorteadas tenha totalizado 1367 crianças, esta se reduziu devido ao abandono escolar (5,9\%), transferência $(5,6 \%)$, faltas no dia da colheita ou da tomada das medidas antropométricas $(4,3 \%)$, à não autorização dos pais ou à idade superior a 120 meses $(3,1 \%)$; falta da data de nascimento $(5,5 \%)$ e recusa pela criança $(0,2 \%)$.

A distribuição da população amostral segundo sexo, idade a escolaridade dos pais é apresentada nas Tabelas 1 e 2 .

TABELA 1. Distribuição da Amostra Estudada Segundo Sexo e Faixa Etária de Alunos Ingressantes nas Escolas Públicas do Município de Osasco, São Paulo, 1991

\begin{tabular}{|c|c|c|c|c|c|c|}
\hline \multirow{3}{*}{ Idade (anos) } & \multicolumn{4}{|c|}{ Sexo } & \multirow{2}{*}{\multicolumn{2}{|c|}{ Total }} \\
\hline & \multicolumn{2}{|c|}{ Masculino } & \multicolumn{2}{|c|}{ Feminino } & & \\
\hline & $\mathrm{N}^{\circ}$ & $\%$ & $\mathrm{~N}^{\circ}$ & $\%$ & $\mathrm{~N}^{\circ}$ & $\%$ \\
\hline $6 \dashv 8$ & 440 & 84,5 & 447 & 87,3 & 887 & 85,9 \\
\hline $8 \dashv 10$ & 81 & 15,5 & 65 & 12,7 & 146 & 14,1 \\
\hline Total & 521 & 100,0 & 512 & 100,0 & 1033 & 100,0 \\
\hline
\end{tabular}


TABELA 2. Distribuição da Amostra Estudada Segundo Escolaridade dos Pais de Alunos Ingressantes nas Escolas Públicas do Município de Osasco, São Paulo, 1991

\begin{tabular}{lrrcrrr}
\hline \hline & \multicolumn{3}{c}{ Pai } & \multicolumn{3}{c}{ Mãe } \\
Escolaridade & \multicolumn{1}{c}{$\mathrm{N}^{\circ}$} & \multicolumn{1}{c}{$\%$} & \%Acumulada & $\mathrm{N}^{\circ}$ & $\%$ & $\%$ Acumulada \\
\hline Analfabeto & 44 & 5,7 & 5,7 & 44 & 4,7 & 4,7 \\
Primário Incompleto & 117 & 15,2 & 20,9 & 121 & 12,9 & 17,6 \\
Primário Completo & 435 & 56,6 & 77,5 & 641 & 68,6 & 86,2 \\
Ginásio Completo & 173 & 22,5 & 100,0 & 129 & 13,8 & 100,0 \\
\hline Total & 769 & 100,0 & & 935 & 100,0 & \\
\hline \hline
\end{tabular}

Primário Completo - cursou as 4 primeiras séries do $1^{\circ}$ grau.

Ginásio Completo - cursou as 8 séries do $1^{\circ}$ grau.

\section{Prevalência de Anemia}

A média dos valores de hemoglobina da amostra estudada situou-se em torno de 11,8 $\mathrm{g} / \mathrm{dl}$ e desvio-padrão de $1,27 \mathrm{~g} / \mathrm{dl}$.

A Tabela 3 mostra a distribuição de crianças segundo valores de hemoglobina.

A Tabela 4 apresenta a prevalência (\%) de anemia (valores de hemoglobina inferiores a $12 \mathrm{~g} / \mathrm{dl}$ ) segundo sexo e idade. $\mathrm{O}$ risco de anemia foi superior entre as crianças que ingressaram no primeiro grau com idade superior a oito anos (RP $=1.22$; IC 95\%: 1,05-1,41). Não se observaram diferenças estatisticamente significantes quanto à distribuição de prevalência por sexo.

\section{Indicadores Antropométricos}

As distribuições dos valores de escore $\mathrm{Z}$ dos índices peso para idade (PR), peso para altura (P/A) apresentaram curvas bem aproximadas à de referência (NCHS). Já a distribuição dos valores do índice altura para idade (AJI) apresentou um deslocamento para a esquerda da curva de referência (Figura 1). A prevalência de valores abaixo de -2 escore $\mathrm{Z}$ relacionada à $\mathrm{A} / \mathrm{I}$ foi de $3,97 \%$. Não foram encontrados valores abaixo de -2 escores $Z$ para P/I e P/A. Os valores de escores $\mathrm{Z}$ de $\mathrm{A} / \mathrm{I}$ entre crianças do sexo masculino e feminino, de todas as idades, apresentaram-se muito semelhantes aos valores para sexos combinados. A distribuição dos valores

TABELA 3. Distribuição da Amostra Estudada Segundo os Valores de Hemoglobina dos Alunos Ingressantes nas Escolas Públicas do Município de Osasco, São Paulo, 1991

\begin{tabular}{lrrr}
\hline \hline Valores de Hemoglobina & & & \\
\hline$<7$ & 3 & 0,3 & 0,3 \\
$7-8$ & 4 & 0,4 & 0,7 \\
$8-9$ & 10 & 1,0 & 1,7 \\
$9-10$ & 33 & 3,2 & 4,9 \\
$10-11$ & 148 & 14,3 & 19,2 \\
$11-12$ & 329 & 31,8 & 51,0 \\
$>12$ & 506 & 49,0 & 100,0 \\
\hline Total & 1033 & 100,0 & \\
\hline \hline
\end{tabular}


TABELA 4. Distribuição dos Anêmicos Segundo Sexo e Idade dos Alunos Ingressantes nas Escolas Públicas do Município de Osasco, São Paulo, 1991

\begin{tabular}{|c|c|c|c|c|c|c|}
\hline \multirow{3}{*}{ Idade (anos) } & \multicolumn{4}{|c|}{ Sexo } & & \\
\hline & \multicolumn{2}{|c|}{ Masculino } & \multicolumn{2}{|c|}{ Feminino } & \multicolumn{2}{|c|}{ Total } \\
\hline & $\%$ & $\mathrm{~N}$ & $\%$ & $\mathrm{~N}$ & $\%$ & $\mathrm{~N}$ \\
\hline $\begin{array}{l:ll}6 & 18\end{array}$ & 48,3 & 213 & 50,6 & 226 & 49,4 & 439 \\
\hline $\begin{array}{l:l}8 & 10\end{array}$ & 65,0 & 52 & 55,4 & 36 & 60,1 & 88 \\
\hline Total & 50,9 & 265 & 51,2 & 262 & 51,0 & 527 \\
\hline
\end{tabular}

FIGURA 1. Escore Z de Altura por Idade 6 a 10 anos, Ambos os Sexos

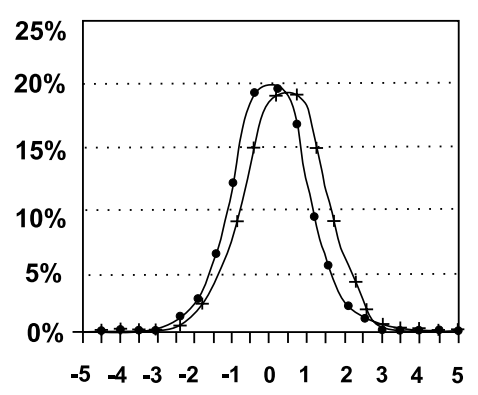

- Observado

+ Referência

Fonte: Osasco, 1991

de escore $\mathrm{Z}$ de $\mathrm{A} / \mathrm{I}$ segundo faixas etárias na amostra estudada, para ambos os sexos, é apresentada nas Figuras 2 e 3.

A curva relativa às crianças com idade superior a 8 anos apresenta um desvio maior à esquerda em relação aos valores da referência do que aquela relativa às crianças com idade inferior a 8 anos. $O$ risco de déficit estatural (valores de escore $Z$ abaixo de -2) é maior nas crianças que estão freqüentando tardiamente a $1^{\mathrm{a}}$ série $(\mathrm{RP}=3,51$, IC 95\% = 1,90 - 6,46).

Não foi encontrada associação significativa entre os anêmicos (indivíduos com $\mathrm{Hb}<12 \mathrm{~g} / \mathrm{dl}$ ) e déficit estatural definido por $Z$ score $<-2$.

A distribuição da prevalência de déficit estatural e de anemia segundo nível de escolaridade do pai ou responsável é apresentada na Tabela 5. A prevalência de déficit estatural entre crianças de pais analfabetos $(9,1 \%)$ foi superior à das demais faixas de escolaridade; porém, não houve associação entre essas duas variáveis $\left(\chi^{2}=3,05 ; \alpha=5 \%\right)$.
A presença de anemia também foi mais elevada em crianças cujos pais eram analfabetos $(63,4 \%)$, man não houve associação entre as duas variáveis $\left(\chi^{2}=3,75 ; \alpha=5 \%\right)$.

FIGURA 2. Encore Z-Score de Altura por Idade 6 a 8 anos, Ambos os Sexos

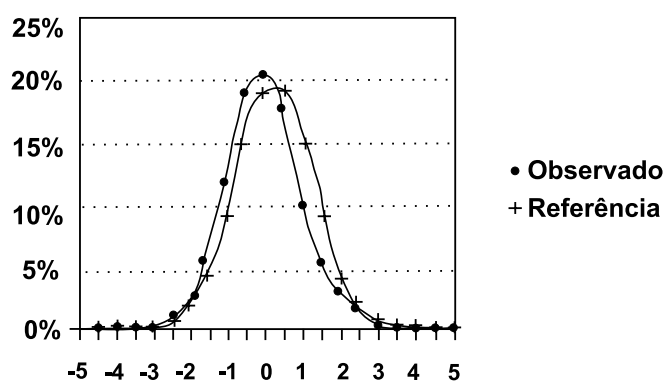

Fonte: Pesquisa de Campo

FIGURA 3. Encore Z-Score de Altura por Idade 8 a 10 anos, Ambos os Sexos

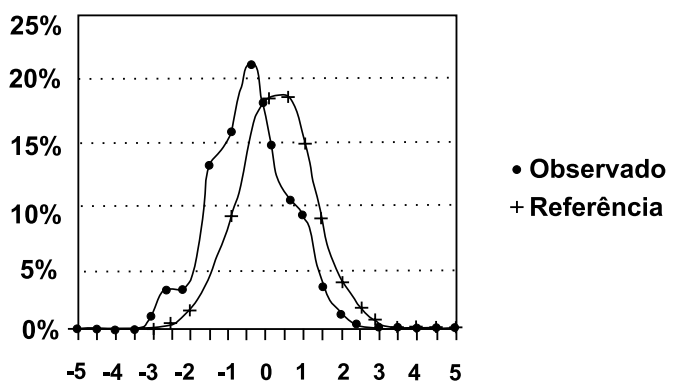

Fonte: Pesquisa de Campo 
TABELA 5. Distribuição Percentual do Déficit Estatural e de Anemia Segundo Escolaridade dos Pais de Alunos Ingressantes das Escolas Públicas do Município de Osasco, São Paulo, 1991

\begin{tabular}{lccc}
\hline \hline Escolaridade do Pai & $(\mathrm{n})$ & $\begin{array}{c}\text { Déficit Estatural (\%) } \\
\leq 2 \text { escore Z }\end{array}$ & $\begin{array}{c}\text { Anemia (\%) } \\
<12 \mathrm{~g} / \mathrm{dl}\end{array}$ \\
\hline Analfabeto & $(44)$ & 9,1 & 63,4 \\
Primário Incompleto & $(117)$ & 5,1 & 47,9 \\
Primário Completo & $(435)$ & 3,7 & 50,8 \\
Ginásio Completo & $(173)$ & 2,9 & 45,1 \\
\hline Total & $(769)$ & 4,0 & 49,8 \\
\hline \hline
\end{tabular}

\section{Distribuição Geográfica da Prevalência de Anemia e do Déficit Estatural}

Com a finalidade de observar a distribuição geográfica dos anêmicos, as escolas foram localizadas segundo as regiões administrativas: Região Central (escolas localizadas nas Regiões AR-3, AR-4, AR-5 e AR-6) e Região Periférica (escolas localizadas nas Regiões AR-1, AR-2, AR-7 e AR-8), conforme Figura 4. A prevalência de anemia apresentada nas escolas da Região Central foi de 41,7\% ( $n=396$ ) e da Região Periférica foi de $56,9 \%(n=637)$.

Houve forte associação entre pertencer à Região Periférica e Anemia $\left(\chi^{2}=3,05 ; \alpha=5 \%\right)$.

A média de valores de hemoglobina para essas regiões consideradas foi de $12,1 \mathrm{~g} / \mathrm{dl}(\mathrm{DP}=1,24)$ para a Região Central e de 11,7 g/dl (DP = 1,34) para a Região Periférica, apresentando diferença significante, para $\mathrm{p}<0,001$ (teste $\mathrm{t}$ ).

Outro dado que mostra importante diferença entre a Região Central e Periférica é que somente 6\% das escolas sorteadas na Região Central apresentaram prevalências de anemia superiores a $60 \%$ enquanto que essa prevalência foi apresentada em 50\% das escolas sorteadas na Região Periférica.

A distribuição da variável "escolaridade do pai", e a região geográfica da escola mostra que a Região Periférica apresenta $7,8 \%$ de pais analfabetos e $18,8 \%$ de pais com primário incompleto, enquanto na Região Central essas percentagens foram de $3,3 \%$ e $11,1 \%$. Houve associação entre pertencer à Região Periférica e ser analfabeto.
FIGURA 4. Prevalência de Anemia (\%) Ingressantes das Escolas de Primeiro Grau de Diferentes Administrações Regionais, Município de Osasco, São Paulo, 1991

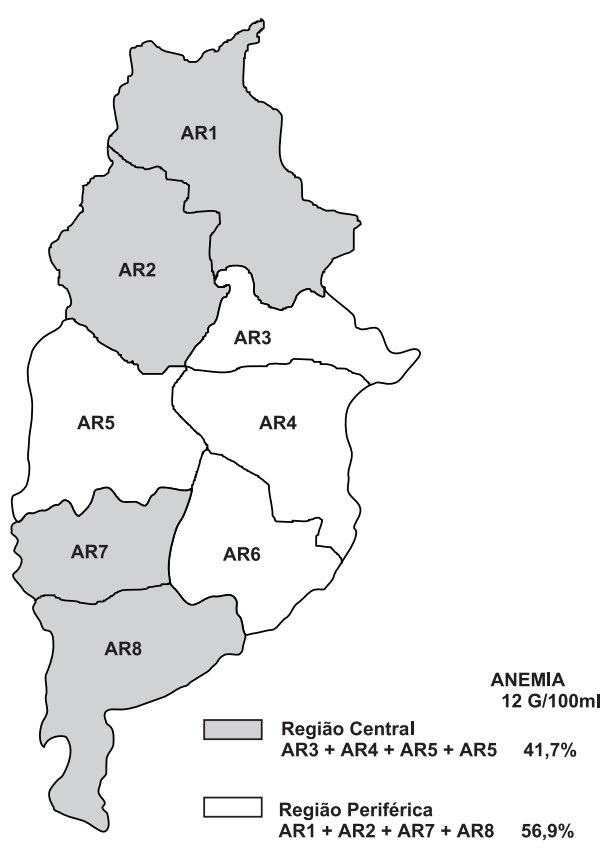

\section{DISCUSSÃO}

Estudos de prevalência de anemia têm mostrado que ém populações não anêmicas, aproximadamente $2 \%$ dos indivíduos apresentam concentração de hemoglobina abaixo dos valores propostos pela OMS, como limites da normalidade. A deficiência de ferro continua prevalente no mundo afetando particularmente ges- 
tantes e crianças. Revisão com base em informações disponíveis na OMS e em centros de documentação para estimar prevalência de anemia por sexo, idade e país, na década de 80 , mostrou prevalência de anemia para a faixa etária de 5 a 12 anos de, aproximadamente $49 \%$ na África, $13 \%$ na América no Norte, $26 \%$ na América Latina, 22\% na Ásia Oriental, 50\% na Ásia Meridional, 5\% na Europa e 15\% na Oceânia (DeMaeyer \& Adiels-Tegman, 1985).

$\mathrm{O}$ presente estudo encontrou a prevalência para a população, de idade entre 6 e 10 anos, de escolares ingressantes em escolas públicas de um Município da Região da Grande São Paulo de $51 \%$, considerando o nível crítico de hemoglobina utilizado para caracterizar a presença de estado anêmico, conforme proposto pela OMS.

A amostra foi representativa das classes de escolares ingressantes, caracterizando grupos de crianças que podem ser consideradas de nível socioeconômico semelhante. A análise mostrou diferenças nas prevalências de anemia segundo localização geográfica. A Região Periférica apresentou níveis de prevalência superiores aos encontrados nas escolas da Região Central (Figura 4), evidenciando que as populações residentes na periferia do município cujas características socioeconômicas são piores do que as da população central, estão mais expostas a esta deficiência, embora sua presença seja importante também na Região Central. A escolaridade do pai foi confirmada como indicador social sensível na detecção da patologia. É uma variável de fácil obtenção, não apresentando problemas de fidedignidade de informação e pode indicar o estrato socioeconômico da família, como experimentado por Monteiro et al. (1986) e sugerido por Zurayk et al. (1987).

A escassez de dados epidemiológicos sobre anemia para esse grupo etário no Brasil dificulta a análise comparativa dos resultados encontrados neste estudo. Os dados disponíveis se reportam, em geral, às crianças menores de 5 anos e às gestantes ou a usuários dos Serviços de Saúde.

Uma questão que parece relevante se refere aos pontos de corte estabelecidos para o diagnóstico de anemia. Se até os 5 anos de idade o ponto de corte do nível de hemoglobina estabelecido pela OMS é de $1 \mathrm{lg} / \mathrm{dl}$, os níveis de 11 a
$12 \mathrm{~g} / \mathrm{dl}$ apresentados em crianças com idades de 6 a 7 anos, devem ser analisados com certo cuidado, pois, a criança com idade entre 7 e 10 anos, também encontra-se em fase de crescimento importante, com aumento correspondente da volemia e a concentração da hemoglobina pode decrescer concomitantemente.

Ressaltados esses aspectos e considerando que os dados preocupantes, ora apresentados, foram obtidos utilizando-se os padrões de referência recomendados, fica evidente a existência do problema de anemia exigindo medidas de prevenção.

Quanto ao estado nutricional, 3,97\% da amostra estudada encontravam-se com desnutrição crônica, isto é, abaixo de - 2 escores $Z$ do indicador altura/idade. Não foi constatada a presença de desnutrição aguda quando utilizado o indicador peso/altura e a presença de déficits de peso para idade também não foi importante. Embora a prevalência de desnutrição crônica esteja próxima do esperado, há evidente deslocamento da curva de distribuição para a esquerda principalmente na das crianças com mais de 8 anos ou que ingressam mais tardiamente na escola (Figura 3 ).

A anemia não esteve associada à presença da desnutrição, repetindo os resultados de outros estudos (Cesar, 1990; Monteiro \& Szarfarc, 1987; Sigulem et al., 1978).

A conseqüência mais óbvia da deficiência de ferro é a anemia com todas as suas seqüelas. Contudo, têm aumentado as evidências de que a deficiência de ferro também traz outros efeitos adversos nos processos metabólicos incluindo o transporte de elétrons, metabolismo de catecolaminas, na síntese do DNA e no sistema enzimático (Baynes \& Bothwell, 1990; Dallman, 1989; Jacobs, 1982).

Existem duas medidas de combate à deficiência de ferro: suplementação e fortificação. A suplementação, feita por meio de fármacos, é recomendada tanto para induzir o aumento dos níveis de hemoglobina em indivíduos já anêmicos como para prevenir anemia em grupos de risco. A fortificação de alimentos melhora o estado nutricional de grupos populacionais e representa o caminho definitivo para resolver o problema da deficiência do ferro. É necessário e urgente encontrar os alimentos mais adequados à tal fortificação, que respeitem os hábitos 
de consumo e que sejam acessíveis à maioria da população brasileira.

Esperava-se encontrar prevalência de anemia importante na faixa etária estudada, uma vez que estudos anteriores haviam observado taxas altas em crianças até 5 anos. Entretanto, os resultados deste estudo superaram as expectativas e ressaltaram a urgência de implantar programas de controle da anemia na população de escolares.

Tendo em vista que a deficiência de ferro na alimentação tem sido apontada como determinante principal da maioria dos casos, revelando-se como a carência nutricional mais prevalente no mundo atual (OMS, 1975; DeMaeyer et al., 1989; INACG, 1977), a merenda escolar torna-se um instrumento da maior importância no controle dessa deficiência. Fortificá-la, aumentar a biodisponibilidade do ferro dos alimentos utilizados nesta refeição e avaliar a eficácia dessa intervenção é o passo que se segue a este diagnóstico.

\section{RESUMO}

STEFANINI, M. L. R.; COLLI, C.; LERNER, B. R.; LEI, D. L. M.; CHAVES, S. P.; DI PIETRO, M. S.; OLIVEIRA, A. A. M. \& SZARFARC, S. C. Anemia e Desnutrição em Escolares da Rede Pública do Município de Osasco, São Paulo, Brasil. Cad. Saúde Públ., Rio de Janeiro, 11 (3): 439447, jul/set, 1995.

Com o objetivo de estimar a prevalência de anemia e conhecer o estado nutricional de escolares, foi realizado um estudo em uma amostra dos alunos ingressantes nas primeiras séries do primeiro grau das escolas públicas de Osasco, município integrante da Região Metropolitana de São Paulo. O diagnóstico de anemia foi feito através da dosagem da concentração de hemoglobina do sangue colhido por punção digital. O nível crítico utilizado para caracterizar a presença de anemia foi o estabelecido pela OMS. O estado nutricional foi avaliado utilizando os indicadores peso/idade e altura/ idade expressos em escores $\mathrm{Z}$ e a população de referência do NCHS. A prevalência, de anemia encontrada foi de $51 \%$. Houve diferença nos níveis de prevalência quanto à localização geográfica das escolas, sendo maior $(56,9 \%)$ na Região Periférica do que na Região Central (41,7\%). A presença de anemia também foi mais elevada em crianças cujos pais eram analfabetos. $\mathrm{O}$ risco de anemia foi superior entre as crianças que ingressaram no primeiro grau com idade superior a 8 anos. Não foi constatada presença significante de desnutrição aguda. Os resultados deste estudo destacam a anemia entre escolares como importante problema de Saúde Pública e ressaltam a urgência da implantação de programas de controle da anemia na população de escolares.

Palavras-Chave: Deficiência de Ferro; Anemia; Anemia Nutricional; Desnutrição; Escolares

\section{REFERÊNCIAS BIBLIOGRÁFICAS}

BAYNES, R. D. \& BOTHWELL, T. H., 1990. Iron deficiency. Annual Reviews of Nutrition, 10: 133-148.

BECKER, N. A. \& LECHTIG, A., 1986. Brasil: Evolução da Mortalidade Infantil no Período 1977-1984. Brasília, DF: Ministério da Saúde.

CESAR, A. T., 1990. O Uso do Ácido Ascórbico no Controle da Deficiência de Fe Utilizando a Estrutura do Programa de Merenda Escolar. Tese de Mestrado, São Paulo: Faculdade de Saúde Pública, Universidade de São Paulo.

DALLMAN, P. R., 1989. Iron deficiency: does it matter? Journal of International Medicine, 226: 367-372.

DeMAEYER, E. \& ADIELS-TEGMAN, M., 1985. The prevalence of anaemia in the world. World Health Statistics Quarterly, 38:302-316.

DeMAEYER, E. M.; DALLMAN, P.R.; GURNEY, J. M.; HALLBERG, L.; SOOD, S. K. \& SRIKANTIA, S. G., 1989. Preventing and Controlling Iron Deficiency Anemia Through Primary Health Care. Genebra: WHO.

INACG (International Nutritional Anemia Consultative Group), 1977. Guidelines for the Eradication of Iron Deficiency. Goteborg: INACG. 
JACOBS, A., 1982. Nom-Praematological effects of iron deficiency clinical. Haematology, 11: 353-364.

JELLIFFE, D. B., 1968. Evaluación del Estado de Nutrición de la Comunidad. Ginebra: OMS.

LIRA, P. I. C.; CARTSAGENA, H. A.; ROMANI, S. A. M.; TORRES, M. A. A. \& BATISTA-FILHO, M., 1985. Estado nutricional de crianças menores de seis anos, segundo posse da terra, em áreas rurais do Estado, nordeste do Brasil. Archivos Utinoamericanos de Nutrición, 35: 247-257.

MONTEIRO, C. A. \& SZARFARC, S. C., 1987. Estudo das condições de saúde das crianças no município de São Paulo, SP (Brasil) 1984-1985. V. Anemia. Revista de Saúde Pública, 21: 255260.

MONTEIRO, C. A.; LEI, D. L. M.; MONDINI, L.; CORDELINI, S.; BARATHO, R. M.; CHAVES, S. P. \& BONALDO, E., 1989. Coleta e Análise da Altura de Escolares em um Sistema de Vigilância Nutricional: Desenvolvimento de Metodologia, Implantação e Avaliação. Relatório Técnico, São Paulo, Faculdade de Saúde Pública, Universidade de São Paulo. (Mimeo.)

MONTEIRO, C. A.; ZUNINGA, H. P. P.; BENICIO, M. H. D. \& SZARFARC, S. C., 1986. Estudo das condições de saúde das crianças no Município de São Paulo, SP (Brasil), 1984 - 1985. I. Aspectos metodológicos, características socioeconômicas e ambiente físico. Revista de Saúde Pública, 20: 435-445.

MOYSES, M. A. A., 1979. Deficiência de Ferro e Desenvolvimento Cognitivo: um Estudo Experimental em Escolares. Tese de Doutorado, São Paulo: Faculdade de Medicina, Universidade de São Paulo.
OMS (Organización Mundial de la Salud), 1975. Lucha Contra la Anemia Nutricional Especialmente Contra la Carencia de Hierro. Genebra: OMS. (Série de Informes Técnicos, 580)

SALZANO, A. C.; TORRES, M. A. A.; BATISTAFILHO, M. \& ROMANI, S. A. M., 1985. Anemia em crianças de dois serviços de saúde de Recife, PE (Brasil). Revista de Saúde Pública, 19: 499-507.

SALZANO, A. C.; BATISTA-FILHO, M.; FLORES, H. \& CALADO, C. L. A., 1980. Prevalência de anemia no ciclo gestacional em dois estados do nordeste brasileiro, Pernambuco e Paraíba. Revista Brasileira de Pesquisas Médicas e Biológicas, 13: 211-214.

SIGULEM, D. M.; TUDISCO, E. S.; GOLDENBERG, P.; ATHAIDE, M. M. M. \& VAISMAN, E., 1978. Anemia ferropriva em crianças do município de São Paulo. Revista de Saúde Pública, 12: 168-178.

SZARFARC, S. C., 1974. Anemia ferropriva em parturientes e recém-nascidos. Revista de Saúde Pública, 8: 369-374.

1985a. Anemia nutricional entre gestantes atendidas em Centros de Saúde do Estado de São Paulo (Brasil). Revista de Saúde Pública, 19: 450-457.

, 1985b. Diagnóstico de deficiência de ferro na infância. Revista de Saúde Pública, 19: 278-284.

WHO (World Health Organization/Working Group), 1986. Use and interpretation of anthropometric indicators of nutritional status. Bulletin of the World Health Organization, 64: 929-941.

ZURAYK, H.; HALABI, S. \& DEEB, M., 1987. Measures of social class based on education for use in health studies in developing countries. Journal of Epidemiology and Community Health, 41: 173-179. 